\title{
Development of an ASIC preamplifier for electromagnetic sensor probes for monitoring space electromagnetic environments
}

\author{
Mitsunori Ozaki ${ }^{*}$, Satoshi Yagitani ${ }^{1}$, Hirotsugu Kojima ${ }^{2}$, Ken Takahashi ${ }^{3}$, Hiroki Koji ${ }^{3}$, Takahiro Zushi ${ }^{2}$ \\ and Yuya Tokunaga'
}

\begin{abstract}
Background: Multipoint observations of plasma waves are essential for separating spatial and temporal variations of a plasma turbulence. Miniaturization and high environmental (temperature and radiation) robustness are key requirements for scientific instrument design toward a sensor network consisting of palm-sized probes. With increasing these demands, a preamplifier for the 3-axis loop antenna of an electromagnetic sensor probe has been developed by using application-specific integrated circuit (ASIC) technology with a $0.25-\mu \mathrm{m}$ complementary metal-oxide-semiconductor process.

Findings: In the present study, a new temperature compensation method is proposed by using the open-loop gain of the ASIC preamplifier with a bandgap reference (BGR) circuit. Usually, the gain is characterized by the closed-loop gain, which is governed by the accuracy of the polysilicon resistances in a chip. The open-loop gain is characterized by the effective transconductance of the ASIC preamplifier, which often has a negative temperature dependence. The temperature dependence of the gain can be dramatically improved by using the temperature-compensated BGR circuit to cancel out the negative dependence of the transconductance. The temperature dependence of the gain was about $-0.01 \mathrm{~dB} /{ }^{\circ} \mathrm{C}$ in the frequency range within the closed-loop bandwidth. On the other hand, the temperature dependence of the gain at $60 \mathrm{kHz}$ operating with the open-loop gain was improved from $-39 \times 10^{-3}$ to $-2.6 \times 10^{-3} \mathrm{~dB} /{ }^{\circ} \mathrm{C}$ by using the temperature-compensated BGR circuit. Moreover, the radiation robustness for the total ionizing dose (TID) level is evaluated by irradiation with gamma rays from cobalt-60. The ASIC preamplifier is not sensitive to TID effects when a thin gate oxide is used. The ASIC preamplifier showed a high radiation tolerance to at least a total ionizing dose level of $400 \mathrm{krad}(\mathrm{Si})$. Finally, the effectiveness of the ASIC preamplifier is evaluated on the basis of a virtual sounding rocket experiment using theoretical calculations of LF standard electromagnetic waves.

Conclusions: Fundamental issues (miniaturization, low-noise performance, and high environmental robustness) are solved by the presented ASIC preamplifier. The success in developing the high robustness ASIC preamplifier leads to a future mission using a lot of palm-sized probes in space.
\end{abstract}

Keywords: Spaceborne ASIC, Loop antenna, Temperature compensation, High environmental robustness

\footnotetext{
${ }^{*}$ Correspondence: ozaki@is.t.kanazawa-u.ac.jp

1 Kanazawa University, Kakuma-machi, Kanazawa 920-1192, Japan

Full list of author information is available at the end of the article
} 


\section{Findings}

\section{Significance of ASIC technology in plasma wave} observations

Plasma wave observations are important for understanding energy transport due to wave-particle interactions and the propagation characteristics in space plasmas. As an innovative design for plasma wave probing, a space sensor network by a monitoring system for space electromagnetic environments (MSEE) has been proposed by Kojima et al. (2010). The main objective of the MSEE is to reveal the spatial distribution of plasma wave turbulence around a target area with a range of several kilometers around artificial objects such as a space station and spacecraft through a number of palm-sized sensor probes. Plasma wave turbulence of this sort was observed as electrostatic and electromagnetic emissions in the vicinity of the Space Shuttle below a frequency of 100 kHz (e.g., Gurnett et al. 1986; Cairns and Gurnett 1991). The MSEE can also be employed to study natural phenomena with their strong spatial inhomogeneities. As an example of the applications in space science, multiple MSEE sensor probes can contribute to understanding nonlinear plasma turbulence on small (Debye and electron) scales within reconnection and shock regions by monitoring spatial inhomogeneity of plasma waves having short wave lengths, which can be sensitive to Doppler effect related to fast plasma motions (Torbert et al. 2014). Another example is localization of plasma wave sources by deploying a distributed sensor network (Constantinescu et al. 2006). Miniaturization of scientific instruments is a key technology for developing a palm-sized MSEE sensor probe. These probes can then be used to measure the plasma wave turbulence in a high spatial distribution. Application-specific integrated circuit (ASIC) technology is one of the best solutions for the advancement of miniaturization technologies. Recently, ASICs for spaceborne instruments for electromagnetic field observations have been actively studied and developed in order to considerably reduce circuit resources (mass, volume, and power) (e.g., DC magnetic field measurements, see Magnes et al. 2008; Sordo-Ibáñez et al. 2015; AC magnetic field measurements, see Rhouni et al. 2013; Ozaki et al. 2014; plasma wave receivers, see Kojima et al. 2010; Fukuhara et al. 2012). The use of ASICs not only serves to reduce the mass and volume, but it also yields a number of analog circuits with almost identical characteristics. MSEE sensor probes can become powerful tools by exploiting ASIC technology. ASIC technology is also suitable for multipoint ground-based observations of natural VLF waves, by the use of a number of tiny instruments having the same electrical properties. (e.g., localization of ionospheric exit point for down-going whistler-mode waves, see Ozaki et al. 2008; MartinezCalderon et al. 2015).

In this study, an ASIC preamplifier for magnetic field observations in the MSEE sensor probe is developed, characterized, and assessed. The ASIC preamplifier is required to have high robustness against temperature and radiation in the harsh space environment. As a first test of high-temperature robustness, gain insensitivity and a wide operational temperature range $\left(-60\right.$ to $\left.+100{ }^{\circ} \mathrm{C}\right)$ are obtained by using an open-loop gain with a temperaturecompensated current source. Moreover, high radiation tolerance is obtained by using a thin gate oxide. Finally, the effectiveness of the ASIC preamplifier for the MSEE sensor probe is evaluated on the basis of theoretical calculations of LF standard electromagnetic waves.

\section{Brief overview of the MSEE sensor probe}

The prototype of an MSEE sensor probe is shown in Fig. 1. Three sets of self-deployable dipole antennas with a tip-totip length of $1.6 \mathrm{~m}$ and of a square-shaped loop antenna (100 turns each) with an area of $0.01-\mathrm{m}^{2}$ are attached. Analog components for the sensor probe consist of preamplifiers for electric fields constructed by discrete analog parts, an ASIC preamplifier for magnetic fields (the present study), and an ASIC waveform receiver (Fukuhara et al. 2012) for probing six components of electromagnetic fields below $100 \mathrm{kHz}$. For digital components, a peripheral interface controller (dsPIC33EP512GP504) containing 4ch AD converters with sampling hold circuits and a wireless communication module (WH-MZ2, Oki Electric Industry Co., Ltd.) are included. All circuit components and a lithium-ion battery as a power supply are installed into the body $(72 \times 72 \times 72 \mathrm{~mm}$ cube). The waveform data for 6 components of the electromagnetic fields are transmitted to a base unit through the wireless communication module at $115.2 \mathrm{kbps}$. The details of the MSEE sensor probe have been described by Zushi et al. (2015).

\section{ASIC preamplifier for probing magnetic fields}

In this study, an ASIC preamplifier for a 3-axis loop antenna is developed and fabricated into a $5 \times 5 \mathrm{~mm}$ chip. The temperature dependence is improved by using the open-loop gain with a temperature-compensated current source. The three-axis loop antenna is orthogonally built in a square around the MSEE probe body. The ASIC preamplifier for the loop antenna has the design shown in Fig. 2. The circuit parameters for the ASIC preamplifier are listed in Table 1 . The ASIC preamplifier consists of a low-noise differential amplifier (62 dB gain) and a gain adjustment amplifier (6 dB gain). The cutoff frequency $\left(1 / 2 \pi C_{1} R_{2}\right.$ and $\left.1 / 2 \pi C_{3} R_{5}\right)$ of both amplifiers is $100 \mathrm{kHz}$, which is the same as that for the ASIC 


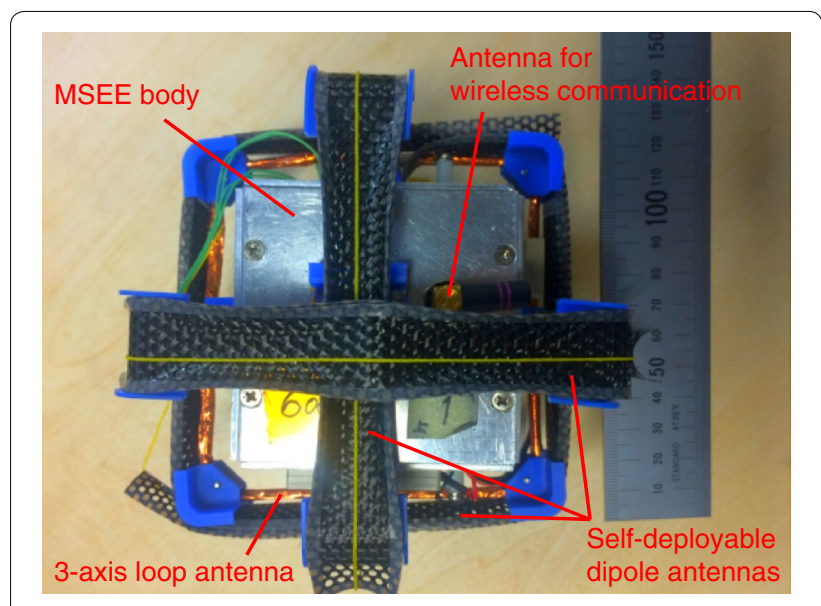

Fig. 1 Photograph of the prototype of an MSEE sensor probe

waveform receiver. Each loop consists of a 100-turn coil with a $0.01-\mathrm{m}^{2}$ area and an electrostatic shield for reducing the capacitive coupling. An external capacitance $C_{0}$ is added to obtain a resonant frequency of $60 \mathrm{kHz}$. Then, magnetic field observations by the MSEE probe give the best noise equivalent magnetic induction (NEMI) at a frequency of $60 \mathrm{kHz}$ for measuring an LF standard electromagnetic wave, which will be discussed later. Wideband NEMI techniques using multiple resonances are discussed in Coillot et al. (2010) and Ozaki et al. (2013). When the external capacitance $C_{0}$ is much larger than the parasitic capacitance (several tens of $\mathrm{pF}$ ) of the coil, the antenna impedance at terminals $v_{\mathrm{inn}}-v_{\mathrm{inp}}$ is given by

$$
Z_{\mathrm{s}}=\frac{R+j \omega L}{\left(1-\omega^{2} L C_{0}+\frac{R}{R_{0}}\right)+j \omega\left(C_{0} R+\frac{L}{R_{0}}\right)},
$$

Table 1 Circuit parameters of the ASIC preamplifier

\begin{tabular}{lll}
\hline Element & Value & Unit \\
\hline$R_{0}$ & 6.0 & $\mathrm{k} \Omega$ \\
$R_{1}$ & 0.80 & $\mathrm{k} \Omega$ \\
$R_{2}, R_{5}$ & 1.0 & $\mathrm{M} \Omega$ \\
$R_{3}$ & 480 & $\mathrm{k} \Omega$ \\
$R_{4}$ & 10 & $\mathrm{k} \Omega$ \\
$C_{0}$ & 3.1 & $\mathrm{nF}$ \\
$C_{1}, C_{3}$ & 1.3 & $\mathrm{pF}$ \\
$C_{2}$ & 0.1 & $\mu \mathrm{F}$ \\
\hline
\end{tabular}

where $R=100 \Omega$ and $L=3 \mathrm{mH}$ are the resistance and inductance of the coil, respectively. Ideally, the antenna impedance approaches infinity at the resonant frequency $f_{\mathrm{r}}=1 / 2 \pi \sqrt{L C_{0}}$, but it is damped by an external resistance $R_{0}=6 \mathrm{k} \Omega$ to avoid the instability caused by the high-quality factor at the resonant frequency. The output noise voltage $e_{\mathrm{o}}$ for an ideal amplifier having a noise gain $G_{\mathrm{n}}$ combined with the loop antenna can be expressed as

$$
e_{\mathrm{o}}=G_{\mathrm{n}} \sqrt{\left(e_{\mathrm{n}}\right)^{2}+\left(i_{\mathrm{n}}\left|Z_{\mathrm{s}}\right|\right)^{2}+4 k_{\mathrm{B}} T \operatorname{Re}\left(Z_{\mathrm{s}}\right)}
$$

where $e_{\mathrm{n}}$ and $i_{\mathrm{n}}$ are the equivalent input noise voltage and current of an ideal amplifier, respectively, $k_{\mathrm{B}}$ is the Boltzmann constant, $T$ is the temperature, and $\operatorname{Re}\left(Z_{\mathrm{s}}\right)$ is the real part of the complex impedance of the loop antenna. The first term in Eq. (2) is usually dominant at $f<f_{\text {r }}$, so the lower voltage noise of the amplifier is well suited to measurements below the resonant frequency. On the other hand, the second term in Eq. (2) is dominant around the resonant frequency because of the high

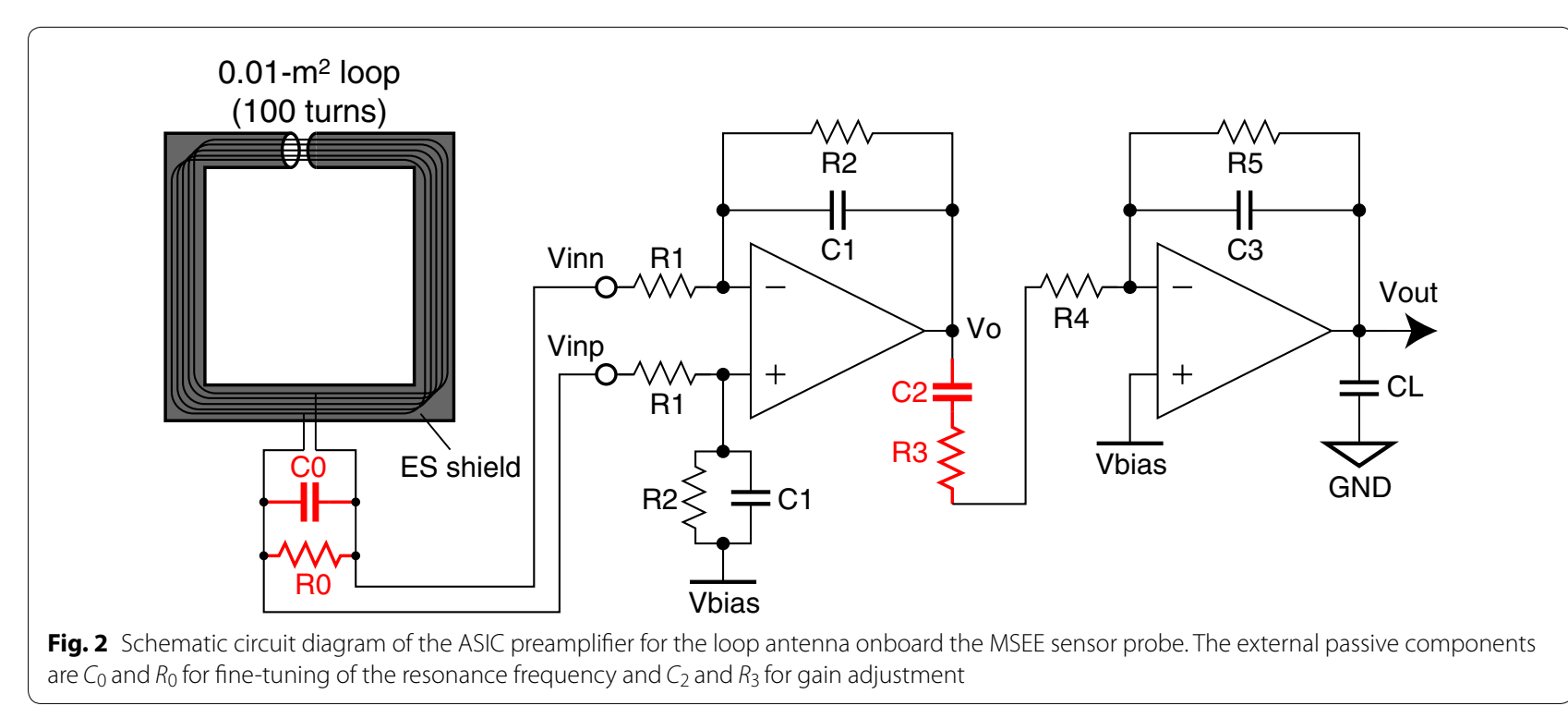


Table 2 Device dimensions for the ASIC preamplifier

\begin{tabular}{lll}
\hline Device & $\boldsymbol{W}(\boldsymbol{\mu m})$ & $\boldsymbol{L}(\boldsymbol{\mu m})$ \\
\hline$M_{0}, M_{11}$ & 800 & 10 \\
$M_{1}, M_{2}$ & 10,000 & 20 \\
$M_{3}, M_{9}, M_{10}, M_{17}, M_{18}$ & 1200 & 10 \\
$M_{4}, M_{5}, M_{12}, M_{13}$ & 2000 & 10 \\
$M_{6}, M_{7}, M_{8}, M_{14}, M_{15}, M_{16}$ & 400 & 10
\end{tabular}

antenna impedance. The current noise for metal-oxidesemiconductor (MOS) transistors is very low compared with that for bipolar junction transistors (BJTs). Thus, complementary metal-oxide-semiconductor (CMOS) technology is more suitable for magnetic field measurements around the resonant frequency (Ozaki et al. 2014).

The complete circuit structure of the ASIC preamplifier is shown in Fig. 3. The dimensions of each MOS transistor are tabulated in Table 2. Our ASIC preamplifier is designed with the standard $0.25-\mu \mathrm{m}$ CMOS process. An ultralow-noise CMOS preamplifier for MSCs has already been developed using ASIC technology (Rhouni et al. 2013; Ozaki et al. 2014). This ASIC preamplifier for the 3-axis loop antenna of an MSEE sensor probe is based on the CMOS preamplifier for MSCs by Ozaki et al. (2014). Large-area $(L W)$ input devices with a high $W / L$ ratio are used to minimize the effects of thermal and flicker noise, where $L$ and $W$ are the channel length and width of a MOS transistor, respectively. The optimization of the device area for a differential amplifier has been discussed by Paulus et al. (2001). The lownoise differential amplifier consists of three stages: (1) a differential amplifier $\left(M_{1}\right.$ and $\left.M_{2}\right)$ with a simple resistive load $\left(R_{\mathrm{d}}=10 \mathrm{k} \Omega\right)$ for achieving sufficiently low noise; (2) a differential amplifier with an active load $\left(M_{4}, M_{5}\right.$, $M_{6}$, and $M_{7}$ ) for improving the open-loop gain; and (3) a voltage buffer having a low output impedance $\left(M_{9}\right.$ and $M_{10}$ ). The gain adjustment amplifier is a typical operational amplifier consisting of an operational transconductance amplifier followed by an output buffer $\left(M_{17}\right.$ and $M_{18}$ ). The output buffer provides rail-to-rail output swings for improving the dynamic range of the ASIC preamplifier. Passive components (resistance and capacitance) use precision polysilicon resistors and metal insulator metal capacitors, which offer relatively high accuracy in electrical performance for the analog circuit design. To achieve a stable operation by improving the phase margin, series resistance $\left(R_{\mathrm{c} 1}, R_{\mathrm{c} 2}\right.$, and $\left.R_{\mathrm{c} 3}\right)$ and capacitance $\left(C_{\mathrm{c} 1}, C_{\mathrm{c} 2}\right.$, and $\left.C_{\mathrm{c} 3}\right)$ compensations are added. Some passive components $\left(R_{3}\right.$ and $\left.C_{2}\right)$ use external parts for fine-tuning of the gain.

\section{Temperature compensation method}

The gain of the ASIC preamplifier in the angular frequency from $1 / C_{2}\left(R_{3}+R_{4}\right)$ to $1 / C_{1} R_{2}\left(=1 / C_{3} R_{5}\right)$ is given by

$$
\begin{aligned}
\left|\frac{v_{\text {out }}}{v_{\text {in }}}\right| & \approx\left|A e_{\mathrm{s}} \cdot H_{1}(\omega) \cdot H_{2}(\omega)\right| \\
& =\left|A e_{\mathrm{s}} \cdot \frac{R_{2}}{\left(Z_{\mathrm{s}}+R_{1}\right)} \cdot \frac{R_{5}}{\left(R_{3}+R_{4}\right)}\right|
\end{aligned}
$$

where

$$
A=\frac{R_{0}}{R_{0}+(R+j \omega L)\left(1+j \omega C_{0} R_{0}\right)}
$$

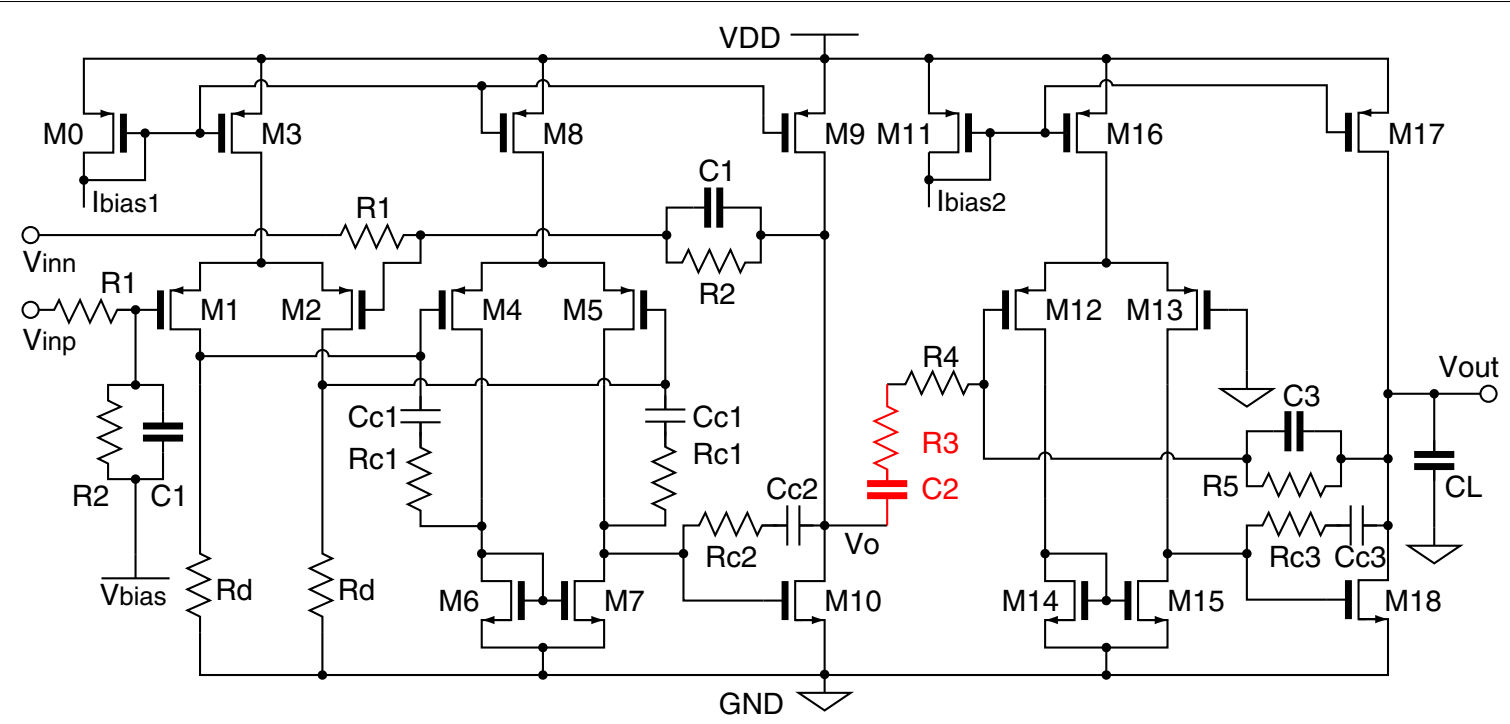

Fig. 3 Integrated circuit structure of the ASIC preamplifier for the MSEE sensor probe 
is a coefficient for voltage dividing, $e_{\mathrm{s}}$ is the induction voltage of the loop, $H_{1}(\omega)$ and $H_{2}(\omega)$ are the transfer functions for the differential amplifier and the gain adjustment amplifier, respectively. The gain of the differential amplifier should be high for low-noise applications, and the closedloop frequency of the gain adjustment amplifier should be larger than the cutoff frequency of $1 / C_{1} R_{2}\left(=1 / C_{3} R_{5}\right)$. The temperature dependence of the differential amplifier is dominant in the ASIC preamplifier, because of its high gain. The output voltage of the differential amplifier can be approximated as follows:

$$
\left|\frac{v_{\mathrm{o}}}{\nu_{\mathrm{in}}}\right| \approx \begin{cases}\left|A e_{\mathrm{s}} \cdot \frac{R_{2}}{Z_{\mathrm{s}}+R_{1}}\right| & \left(\omega \ll \omega_{\mathrm{CL}}\right) \\ A e_{\mathrm{s}} \cdot g_{\mathrm{meff}} R_{\mathrm{eff}} \cdot \frac{1}{\left(1+j \frac{\omega}{\omega_{\mathrm{p} 1}}\right)} \mid & \left(\omega_{\mathrm{CL}} \ll \omega<\frac{1}{C_{1} R_{2}}\right)\end{cases}
$$

where $\omega_{\mathrm{CL}}$ is the closed-loop angular frequency of the differential amplifier, $g_{\text {meff }}$ is the effective transconductance, $R_{\text {eff }}$ is the effective output resistance, and $\omega_{\mathrm{p} 1}$ is the firstpole frequency of the differential amplifier. The effective transconductance is given by $g_{\mathrm{meff}}=g_{\mathrm{m} 1} g_{\mathrm{m} 4} g_{\mathrm{m} 10}$, where $g_{\mathrm{m} 1}, g_{\mathrm{m} 4}$, and $g_{\mathrm{m} 10}$ are the transconductances of the $M_{1}$, $M_{4}$, and $M_{10}$ transistors, respectively. The closed-loop gain of the ASIC preamplifier depends on the accuracy of polysilicon resistors on a chip, as shown in Eq. (6). The size ratio accuracy of polysilicon resistors on the layout is high, but their absolute accuracy is usually poor, owing to the large parameter drift with temperature. The temperature dependence is strongly influenced by the accuracy of the passive components, which may cause a large drift in the electrical performance of the ASIC preamplifier with temperature variation. On the other hand, greater control of the temperature dependence of the transconductance is possible by using the open-loop gain in the frequency range above the closed-loop frequency. The temperature dependence of the transconductance of the $i$ th MOS transistor is given by

$$
\begin{aligned}
\frac{\mathrm{d} g_{\mathrm{m} i}}{\mathrm{~d} T} & =\frac{\partial g_{\mathrm{m} i}}{\partial \mu} \frac{\partial \mu}{\partial T}+\frac{\partial g_{\mathrm{m} i}}{\partial I_{\mathrm{D}}} \frac{\partial I_{\mathrm{D}}}{\partial T} \\
& =\frac{C_{\mathrm{ox}} I_{\mathrm{D}}}{g_{\mathrm{m} i}} \frac{\partial \mu}{\partial T}+\frac{C_{\mathrm{ox}} \mu}{g_{\mathrm{m} i}} \frac{\partial I_{\mathrm{D}}}{\partial T} .
\end{aligned}
$$

where $\mu$ is the mobility, $I_{\mathrm{D}}$ is the drain current, and $C_{\mathrm{ox}}$ is the oxide capacitance. The mobility usually shows a negative temperature dependence $(\partial \mu / \partial T<0)$, so a current source having a positive temperature dependence $\left(\partial I_{\mathrm{D}} / \partial T>0\right)$ could be canceling out the temperature dependence of the transconductance. A sufficiently weak temperature dependence is obtained by using a current source having a positive temperature dependence. In this study, a bandgap reference (BGR) circuit is used to keep the variation in transconductance with temperature to a minimum.

Figure 4 shows the integrated circuit structure of the BGR circuit using the base emitter diodes of lateral BJTs. The device dimensions are listed in Table 3. The BGR circuit consists of a proportional to absolute temperature (PTAT) current generator and a current reference. In Fig. 4, a start-up circuit $\left(M_{\mathrm{a}}, M_{\mathrm{b}}\right.$, and $\left.M_{\mathrm{c}}\right)$ is included to avoid a zero current state. The principle of a PTAT current generator is based on the stable temperature dependence of a differential voltage between two groups of diodes $\left(Q_{1}: Q_{2}\right)$ with different current densities. The PTAT current is given by

$$
I_{\text {PTAT }}=\frac{k_{\mathrm{B}} T}{q R_{\mathrm{a}}} \ln (n),
$$

where $q$ is the elementary charge and $n$ is the ratio of the number of base emitter diodes of lateral BJTs $\left(Q_{1}: Q_{2}=1: n\right)$. The reference currents for the ASIC preamplifier are generated by copying the PTAT current with the current mirror circuits $\left(M_{\mathrm{i}}, M_{\mathrm{j}}\right.$, and $\left.M_{\mathrm{k}}\right)$. It is possible to cancel out the negative temperature dependence of the mobility by using the PTAT current source. In this study, the PTAT current source is designed to have a thermal gradient of $+0.67 \mu \mathrm{A} /{ }^{\circ} \mathrm{C}$ with a $3.3-\mathrm{V}$ supply.

\section{Radiation tolerant layout}

The ASIC preamplifier for spaceborne systems requires tolerance to a harsh radiation environment. The following three influences of radiation on CMOS circuits are well known (e.g., Schrimpf and Fleetwood 2004; Velazco et al. 2007; Cressler and Mantooth 2012): (1) single-event effects due to a single strike by mainly high-energy ions; (2) displacement damage dose effects caused by collisions between neutral particles and silicon nuclei; (3) total ionizing dose (TID) effects due to long-term ionizing radiation damage by low-energy ions and electrons. In analog circuits, the emphasis would be on the TID tolerance, rather than other effects, because the electrical performance would be gradually degraded by the TID level. Moreover, one other distinct type of degradation to the electrical performance of BJTs at a low dose rate is thought to be enhanced low dose rate sensitivity (Hjalmarson et al. 2003), which is negligible for CMOS circuits. The TID tolerance for scientific instruments onboard satellites is usually improved by adding a shield composed of a heavy metal such as Ta or Mo, but this requires a large mass. The TID effect is caused by electron-hole pair production in the gate oxide layer under radiation exposure (Oldham and McLean 2003). Holes become trapped in lattice defects in the gate oxide layer because of their low mobility compared with that of electrons. Accumulated holes are equivalent to a fixed 


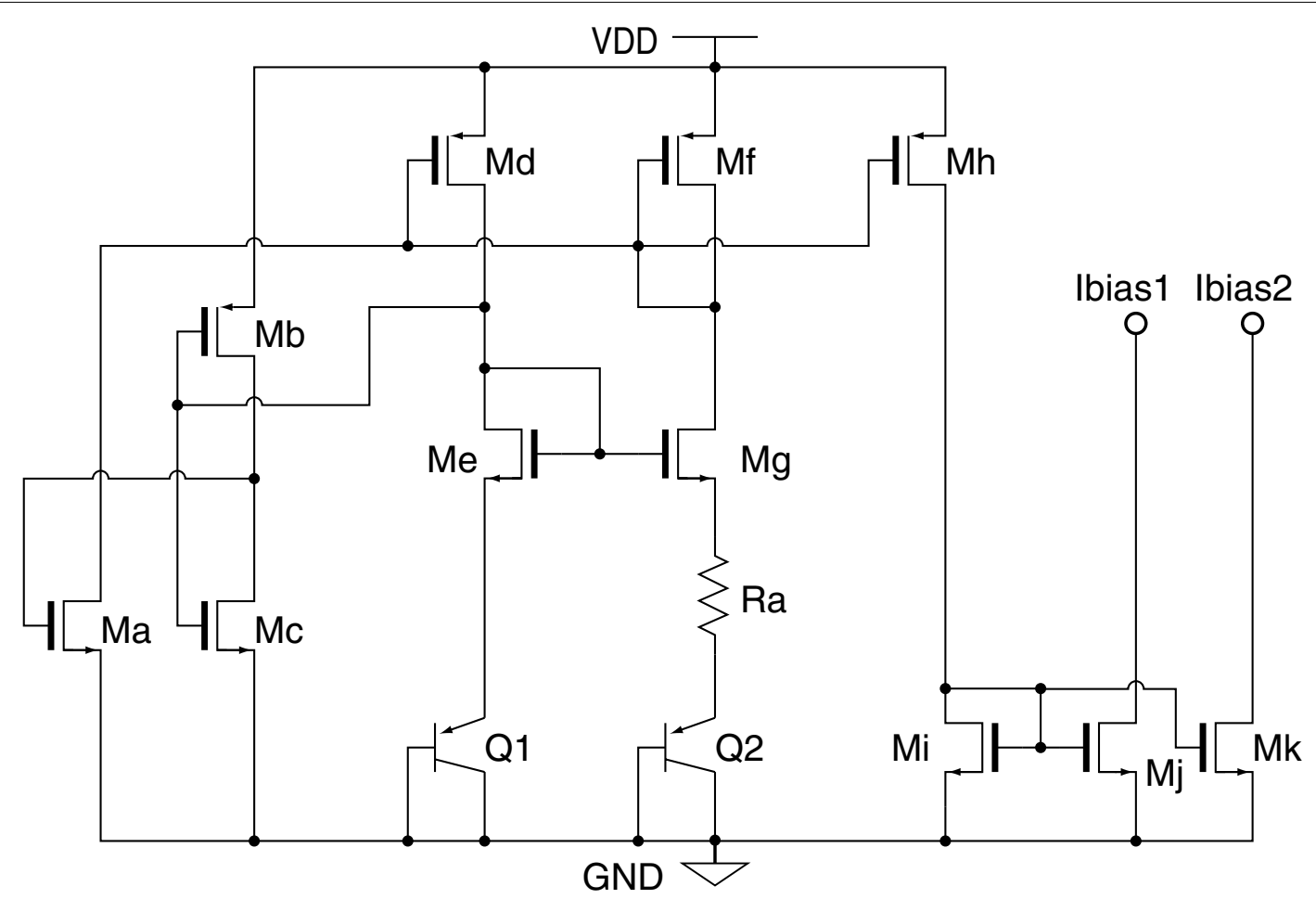

Fig. 4 Circuit structure of the temperature-compensated bandgap reference for the ASIC preamplifier

positive charge. As a result, the threshold potential of the device shifts, and hence, electrical performance parameters such as gain, noise, and consumption current, are degraded. MOS transistors having thin gate oxides can improve the TID tolerance, because thin gate oxides provide a high probability of annihilation for trapped holes and of compensation by tunneling electrons. Thus, ASIC technology is only one solution for ensuring a sufficient TID tolerance without additional mass budget. In this study, MOS devices having thin gate oxides are selected. Additionally, double guard rings surrounding each circuit block are employed to suppress latch-up caused by energetic ions (Voldman 2007).

\section{Results and discussion Gain and NEMI}

Figure 5 shows an optical micrograph of the manufactured ASIC preamplifier. Four sets of preamplifiers and temperature-compensated BGR circuits are built in a $5 \times 5 \mathrm{~mm}$ bare chip. (The fourth circuits are the spares for the 3-axis loop antenna.) The differential amplifier, gain adjustment amplifier, and BGR circuit for each axis occupy $1500 \times 970 \mu \mathrm{m}, 630 \times 630 \mu \mathrm{m}$, and $160 \times 280 \mu \mathrm{m}$, respectively. To suppress the crosstalk between the loop antennas, all circuit components are isolated from each other with an analog ground. The gain measurement and simulation (SPICE) results for the ASIC preamplifier combined with a $0.01-\mathrm{m}^{2}$ loop antenna (100 turns) are plotted in Fig. 6. The measurements were taken with a Helmholtz coil in a magnetically shielded room. The gain was designed to take a resonance frequency of $60 \mathrm{kHz}$ for the detection of an LF standard electromagnetic wave by a virtual sounding rocket experiment, which will be discussed later. The gain measurement results agreed well with the simulation results. The measurements and simulation results for the NEMI of the ASIC preamplifier are plotted in Fig. 7. The NEMI measurement result is almost the same as that obtained by simulation, with the difference being caused by the error in the SPICE parameters and by neglecting the effect of noise due to the drain resistances $R_{\mathrm{d}}$ in the simulation. The NEMI for a previous preamplifier (assembled with discrete electronic components by Yagitani et al. 2011) combined with the 0.01$\mathrm{m}^{2}$ loop (15 turns) is also plotted in Fig. 7. The power consumption of the ASIC preamplifier for a 1-axis loop antenna was $5.0 \mathrm{~mW}$ with a $3.3-\mathrm{V}$ supply, which is half that of the previously reported preamplifier. The NEMI curve of the ASIC preamplifier was designed to be similar to that of the previous preamplifier. The number of coil turns (100 turns) was modified from the previous prototype (15 turns) in order to satisfy the mechanical requirements for the loop antenna in a sounding 


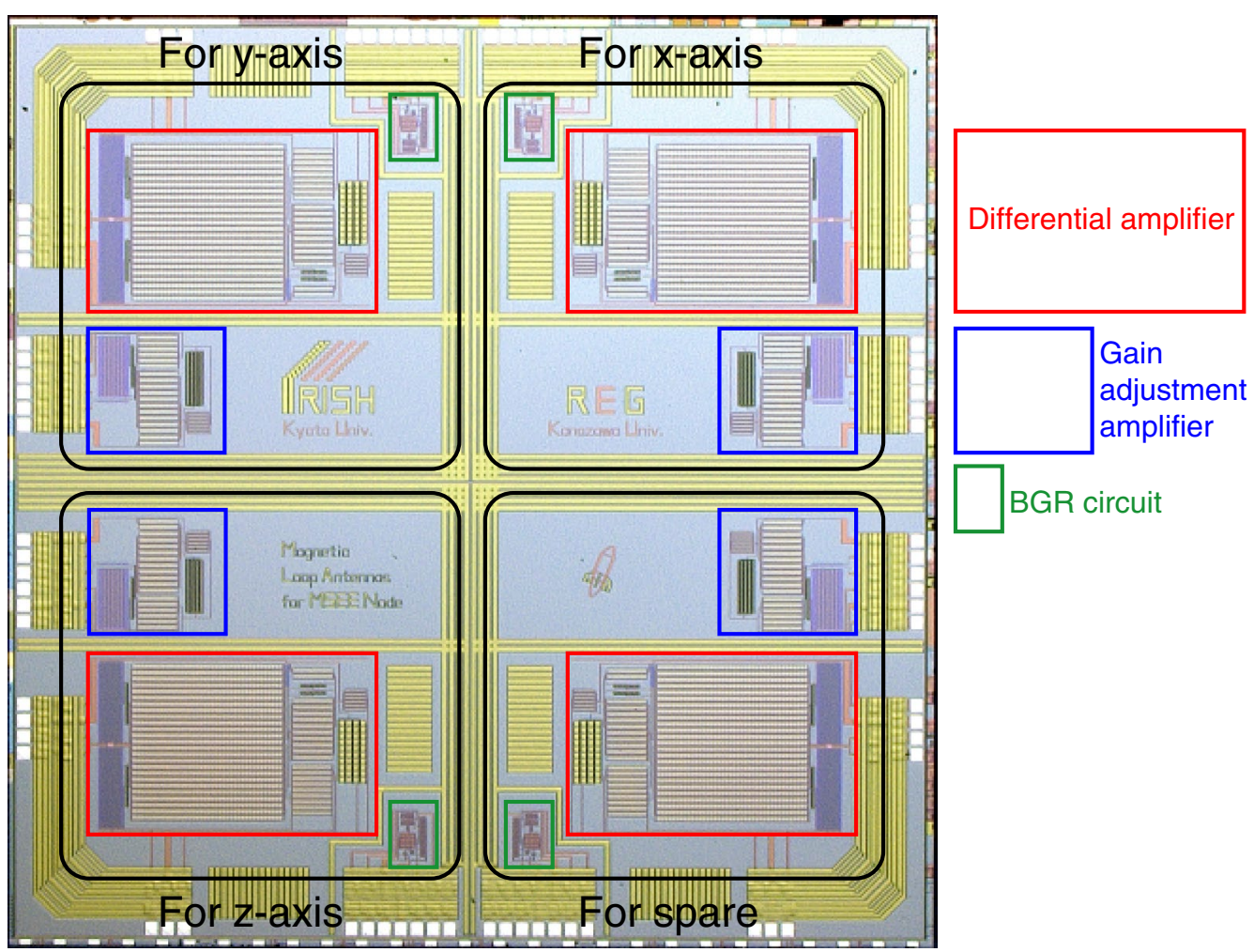

Fig. 5 Optical micrograph of the ASIC preamplifier for the MSEE sensor probe. The chip size is $5 \times 5 \mathrm{~mm}$. The dimensions of the differential amplifier, gain adjustment amplifier, and BGR circuit for each axis are $1500 \times 970 \mu \mathrm{m}, 630 \times 630 \mu \mathrm{m}$, and $160 \times 280 \mu \mathrm{m}$, respectively

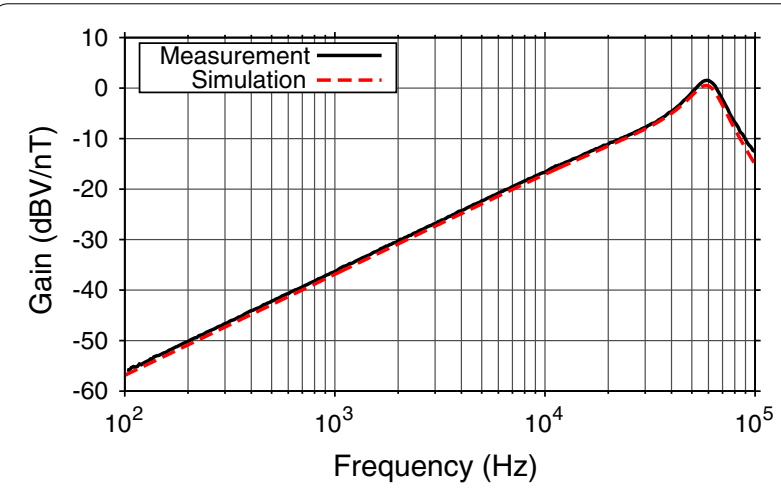

Fig. 6 Measurement and simulation results for the gain of the ASIC preamplifier combined with a $0.01-\mathrm{m}^{2}$ loop

rocket experiment. The equivalent input noise voltage of the ASIC preamplifier is acceptable even though it is $6.7(=100 / 15)$ times worse. The noise characteristics of the ASIC preamplifier can be improved by using larger differential gate inputs (e.g., Rhouni et al. 2013; Ozaki et al. 2014). The NEMI for the ASIC preamplifier is sufficiently low for the detection of whistler-mode emissions
Table 3 Device dimensions and circuit parameters for the BGR circuit

\begin{tabular}{lll}
\hline Device & $\boldsymbol{W}(\boldsymbol{\mu m})$ & $\boldsymbol{L}(\boldsymbol{\mu m})$ \\
\hline$M_{\mathrm{a}}, M_{\mathrm{c}}, M_{\mathrm{e}}, M_{\mathrm{g}}$ & 15 & 1 \\
$M_{\mathrm{b}}$ & 3 & 1 \\
$M_{\mathrm{d}}, M_{\mathrm{f}}$ & 120 & 1 \\
$M_{\mathrm{h}}$ & 720 & 1 \\
$M_{\mathrm{i}}$ & 30 & 1 \\
$M_{\mathrm{j}}, M_{\mathrm{k}}$ & 150 & 1 \\
Device & Value & Unit \\
$Q_{1}: Q_{2}$ & $1: 8$ & \\
$R_{a}$ & 9.11 & $k \Omega$ \\
\hline
\end{tabular}

excited by an electron beam from the Space Shuttle (Gurnett et al. 1986) shown on the same graph as a reference. Also, this MSEE sensor probe will be able to detect large amplitude whistler waves (up to several nT) in the radiation belts with a sufficient NEMI (Cattell et al. 2008). The formation and evolution of coherent whistler waves play an important role in understanding nonlinear 
Table 4 Temperature coefficients of the ASIC preamplifier gain for each frequency

\begin{tabular}{|c|c|c|c|c|}
\hline \multirow[b]{2}{*}{$\begin{array}{l}\text { Frequency } \\
(\mathrm{Hz})\end{array}$} & \multicolumn{2}{|c|}{ Measurements } & \multicolumn{2}{|l|}{ Simulations } \\
\hline & $\begin{array}{l}\text { Without / } \\
\left(\times 10^{-3}\right. \\
\left.\mathrm{dB} /{ }^{\circ} \mathrm{C}\right)\end{array}$ & $\begin{array}{l}\text { With } /_{\text {PTAT }} \\
\left(\times 10^{-3}\right. \\
\left.\mathrm{dB} /{ }^{\circ} \mathrm{C}\right)\end{array}$ & $\begin{array}{l}\text { Without } /_{\text {PTAT }} \\
\left(\times 10^{-3}\right. \\
\left.\mathrm{dB} /{ }^{\circ} \mathrm{C}\right)\end{array}$ & $\begin{array}{l}\text { With } /_{\mathrm{PTAT}} \\
\left(\times 10^{-3}\right. \\
\left.\mathrm{dB} /{ }^{\circ} \mathrm{C}\right)\end{array}$ \\
\hline $1 \mathrm{k}$ & -8.3 & -9.1 & -5.7 & -5.6 \\
\hline $10 k$ & -12 & -8.6 & -7.1 & -5.3 \\
\hline $60 k$ & -39 & -2.6 & -22 & +0.46 \\
\hline
\end{tabular}

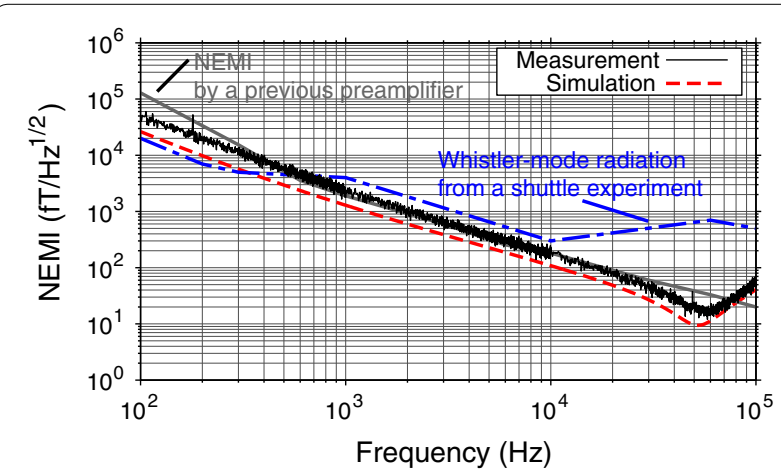

Fig. 7 Measurement (black solid line) and simulation (red dotted line) results for the NEMI for the ASIC preamplifier combined with a 0.01$\mathrm{m}^{2}$ loop (100 turns). The gray solid line is for the NEMI by a previous preamplifier combined with $0.01-\mathrm{m}^{2}$ loop (15 turns) by Yagitani et al. (1994). The dot-dashed line represents the spectral characteristics of the whistler-mode radiation from a Space Shuttle experiment by Gurnett et al. (1986)

wave-particle interactions. The validity of the gain and NEMI for the ASIC preamplifier will be discussed below, on the basis of a virtual sounding rocket experiment.

\section{Temperature test results}

As shown in Fig. 7, the plasma waves radiating from the Space Shuttle experiment had a frequency of over $10 \mathrm{kHz}$. The closed-loop frequency of the preamplifier was designed to be $10 \mathrm{kHz}$ to improve the temperature dependence in the target frequency band $(10-100 \mathrm{kHz})$. Tests for the ASIC preamplifiers were conducted in the temperature range from -60 to $+100{ }^{\circ} \mathrm{C}$ to evaluate the temperature sensitivity of the gain without/with the BGR circuit in a thermostatic chamber (MC-712, ESPEC Corp.). The temperature dependence of the ASIC preamplifier was evaluated by the gain without the loop antenna, because of electromagnetic noise from the facility. Figure 8 shows the measurement (panels a and c) and simulation (panels $b$ and $d$ ) results of the gain profile for each temperature. The temperature behavior without/with the BGR circuit shows a reasonable agreement

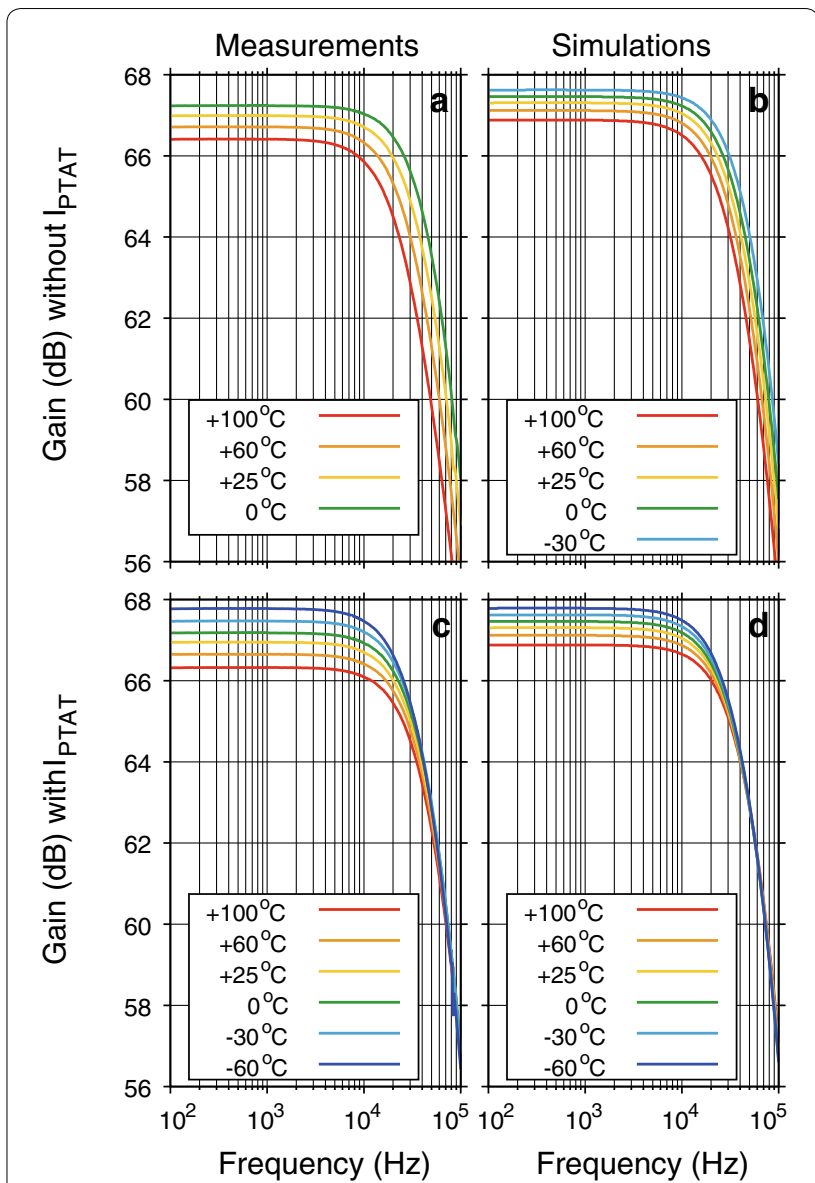

Fig. 8 Measurement and simulation results for the temperature dependence of the gain of the ASIC preamplifier. $\mathbf{a}, \mathbf{c}$ are for the measurements, and $\mathbf{b}, \mathbf{d}$ are for the simulations. $\mathbf{a}, \mathbf{b}$ are the temperature test results without the temperature-compensated BGR circuit, and $\mathbf{c}$, $\mathbf{d}$ are for using the temperature-compensated BGR circuit

between the measurements and the simulations. The ASIC preamplifier was operated in the temperature range from -30 to $+100{ }^{\circ} \mathrm{C}$ without the temperature-compensated BGR circuit in the simulations (panel b), but the operational temperature range was 0 to $+100{ }^{\circ} \mathrm{C}$ in the measurements (panel a). The reason of small errors would be caused by the inaccuracy in the SPICE parameters related to the temperature drift. On the other hand, the operational temperature range was improved from -60 to $+100^{\circ} \mathrm{C}$ by using the temperature-compensated BGR circuit for the measurements and simulations (panels $\mathrm{c}$ and d). It is clearly seen that the temperature-compensated BGR circuit can be effective above $10 \mathrm{kHz}$. The temperature dependence of the gain showed a linearly decreasing trend in units of $\mathrm{dB}$. The temperature coefficients of the gain without/with the BGR circuit are listed in Table 4 for each frequency. The temperature coefficients of the gain below $10 \mathrm{kHz}$ were about $-12 \sim-8 \times 10^{-3} \mathrm{~dB} /{ }^{\circ} \mathrm{C}$ without/with the BGR circuit for the measurements 
and $-7 \sim-5 \times 10^{-3} \mathrm{~dB} /{ }^{\circ} \mathrm{C}$ for the simulations, respectively. These values are almost the same as those for the ASIC preamplifier for MSCs (Rhouni et al. 2013). In the frequency range within the closed-loop bandwidth, the temperature coefficients cannot be controlled, because they are determined by the thermal drift of the polysilicon resistors embedded in the chip, as shown in Eq. (6). They are not inferior in quality, but the temperature coefficients of the gain were dramatically improved by using the BGR circuit. Then, the values reach to -2.6 and +0.46 $\times 10^{-3} \mathrm{~dB} /{ }^{\circ} \mathrm{C}$ for the measurement and the simulation. The experimental temperature coefficient of the gain at $60 \mathrm{kHz}$ with the BGR circuit was equal to one-fifteenth that without the BGR circuit. Other ASIC preamplifiers showed exactly the same insensitivity to temperature. This high resistance to temperature eliminates the need for a calibration process for temperature and improves the reliability of the gain difference with multiple observations by MSEE sensor probes. The frequency range for this temperature compensation method can be improved by decreasing the closed frequency band. The present method using the open-loop gain with the BGR circuit is effective in improving the temperature dependence of the ASIC preamplifier.

\section{Total dose test results}

In order to estimate the sensitivity to the TID effect, TID radiation tests for the ASIC preamplifiers were conducted with gamma rays from cobalt-60 at the Radioisotope Research Center, Tokyo Institute of Technology. The target level of radiation tolerance is usually selected as a maximum TID level of $100 \mathrm{krad}(\mathrm{Si})$ for a satellite experiment. The typical dose rate in space is below $10^{-3} \mathrm{rad} / \mathrm{s}$, but the dose rate used in laboratory experiments is several orders of magnitude higher to reduce the test period. Recommended dose rates for laboratory experiments have been reported elsewhere (e.g., Fleetwood and Eisen 2003 and references therein). The ASIC preamplifiers were irradiated at dose rates of $0.39 \mathrm{rad}(\mathrm{Si}) / \mathrm{s}$ [below $200 \mathrm{krad}(\mathrm{Si})], 0.56 \mathrm{rad}(\mathrm{Si}) / \mathrm{s}$ [200-300 $\mathrm{krad}(\mathrm{Si})]$, and $0.75 \mathrm{rad}(\mathrm{Si}) / \mathrm{s}$ [300-400 $\mathrm{krad}(\mathrm{Si})]$. The TID radiation results were evaluated with the output noise at 40,100, 200,300 , and $400 \mathrm{krad}(\mathrm{Si})$. Here, only the measurement result for $400 \mathrm{krad}(\mathrm{Si})$ irradiation is described, because the measurement results for other TID levels were the same. Pre- and post-radiation results for the equivalent input noise of the ASIC preamplifier are shown in Fig. 9. No difference was detected between the pre- and post-radiation results. Other channels and chips of the ASIC preamplifier showed the same insensitivity under $400 \mathrm{krad}(\mathrm{Si})$ irradiation for the flicker and thermal noise behaviors. The power consumption was also the same for the pre- and post-radiation tests. The equivalent input voltage noise level of the ASIC preamplifier changes with the drain current of each transistor (Rhouni et al. 2013; Ozaki et al. 2014) controlled by the bias currents from the temperature-compensated BGR circuit $\left(I_{\text {bias1 }}\right.$ and

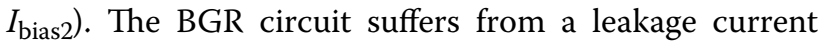
induced by the radiation damage in the transistor diodes (Cao et al. 2013). The variation of bias currents was an increase of $1 \%$ at the room temperature before and after the radiation tests from 24 samples. The results of no difference in the noise level indirectly show that the variation of bias currents causing the degradation of the noise level did not occur after the radiation tests, even though the increase of $1 \%$ in the bias currents was included. It suggests that the BGR circuit has no significant damage from the radiation at the TID level of $400 \mathrm{krad}(\mathrm{Si})$. Thus, it is concluded that the ASIC preamplifier satisfactorily meets the high TID levels of space-quality ASICs.

\section{Virtual sounding rocket experiment}

This section presents a virtual sounding rocket experiment aimed at performing magnetic field observations by an MSEE sensor probe. An LF standard electromagnetic wave radiating from the ground station is one of the observation targets to assess the electrical performance of the MSEE sensor probe. In this study, a virtual sounding rocket experiment for an LF standard electromagnetic wave is performed by using full-wave analysis (Nagano et al. 1975). The calculation model assumes the horizontally stratified ground-free space-ionosphere system with Cartesian coordinates. The $z$-axis represents the altitude direction. The $x$ - and $y$-axes point to the geomagnetic east and north, respectively. The $y-z$ plane is the geomagnetic meridian plane. The plasma in the ionosphere is assumed to be magnetized cold plasma without thermal motion of electrons. The ground is assumed to be an isotropic conductive medium. A dipole source is oriented vertically on the ground. The inhomogeneity of each medium is considered only in the $z$ direction. On the basis of the above assumptions, a spherical wave field $\boldsymbol{e}$ at an arbitrary point $(x, y, z)$ radiating from a dipole source can be expressed as

$\boldsymbol{e}(x, y, z)=\int_{-\infty}^{+\infty} \int_{-\infty}^{+\infty} \tilde{\boldsymbol{e}}\left(k_{x}, k_{y}, z\right) \cdot e^{-j\left(k_{x} x+k_{y} y\right)} \mathrm{d} k_{x} \mathrm{~d} k_{y}$

where $\tilde{\boldsymbol{e}}$ is an elementary plane wave, and $k_{x}$ and $k_{y}$ are the $x$ and $y$ components of the wave normal vector $\boldsymbol{k}$, respectively. Equation (9) indicates that a spherical wave field can be represented as the superposition of a large number of elementary plane waves propagating in all directions of the wave normal vector. Here, the elementary plane waves at an arbitrary altitude $z$ are rigorously calculated with various incident angles in the horizontally 
stratified medium as the full-wave solution. The elementary plane wave in each stratified layer, as established by Nagano et al. (1975), is given by

$$
\frac{\mathrm{d} \tilde{\boldsymbol{e}}}{\mathrm{d} z}=-j k_{0} \boldsymbol{T} \tilde{\boldsymbol{e}},
$$

where $k_{0}$ is the free space wave number and $\boldsymbol{T}$ is a propagation matrix determined by the medium constants (electron density, collision frequency, geomagnetic field, permittivity, and permeability) of each stratified layer. Each elementary plane wave is connected with those in the adjacent layers to satisfy boundary conditions based on Snell's law. The calculation techniques for the superposition of elementary plane waves based on the fullwave solutions have been described in detail by Nagano et al. (1993), Yagitani et al. (1994), and Ozaki et al. (2009). In this study, full-wave analysis is performed on the basis of the study by Nagano et al. (1993) to evaluate the Omega signals radiating from a dipole source on the ground. The infinite integral of Eq. (9) can be numerically computed as a finite integral, because the elementary plane waves become evanescent waves for large $k_{x}$ and $k_{y}$. It is possible to rigorously evaluate the propagation of an LF standard electromagnetic wave in the ionosphere by using full-wave analysis.

The altitude profiles of the electron density and the effective collision frequency between electrons and neutral particles in the daytime and nighttime ionosphere are shown in Fig. 10. The electron densities are taken from the international reference ionosphere model for a northern hemisphere midlatitude (Bilitza 2001). The collision frequency is calculated as proportional to the atmospheric pressure with the MSIS-E-90 model (Hedin 1991). The other calculation parameters are listed in Table 5 . These parameters assume an LF standard electromagnetic wave radiating from Hagane-yama LF Standard Time and Frequency Transmission Station, which is one of Japan's standard electromagnetic wave stations.

Figure 11 shows the daytime (panels a and $b$ ) and nighttime (panels $\mathrm{c}$ and $\mathrm{d}$ ) intensity distributions of LF standard electromagnetic waves on the magnetic meridian $(y-z)$ plane. The electromagnetic waves above the dipole source showed null fields and a figure-of-eight radiation pattern within a horizontal distance of $200 \mathrm{~km}$. The spherical electromagnetic waves in the Earth-ionosphere waveguide showed interference between radiated waves from the dipole source and reflected waves (L-mode) from the lower ionosphere. The interference pattern was particularly clear in the nighttime because of the higher reflection coefficient at the lower ionosphere. In the ionosphere, the LF standard electromagnetic waves propagate as whistler-mode waves along the geomagnetic field line and show an asymmetric distribution in the
Table 5 Calculation parameters for full-wave analysis

\begin{tabular}{lll}
\hline Parameter & Value & Unit \\
\hline Wave frequency & 60 & $\mathrm{kHz}$ \\
Geomagnetic dip angle & 48.2 & degrees \\
Gyrofrequency & 1.271 & $\mathrm{MHz}$ \\
Altitude of dipole source & 900 & $\mathrm{~m}$ \\
Length of dipole source & 200 & $\mathrm{~m}$ \\
Effective radiation power & 25 & $\mathrm{~kW}$ \\
Conductivity of the ground & $1.0 \times 10^{-3}$ & $\mathrm{~S} / \mathrm{m}$ \\
Relative permittivity of the ground & 10 & \\
Altitude range of the ionosphere & 60 to 150 & $\mathrm{~km}$ \\
Altitude resolution & 0.25 & $\mathrm{~km}$ \\
Horizontal distance range & -512 to +511 & $\mathrm{~km}$ \\
Horizontal distance resolution & 1.0 & $\mathrm{~km}$ \\
\hline
\end{tabular}

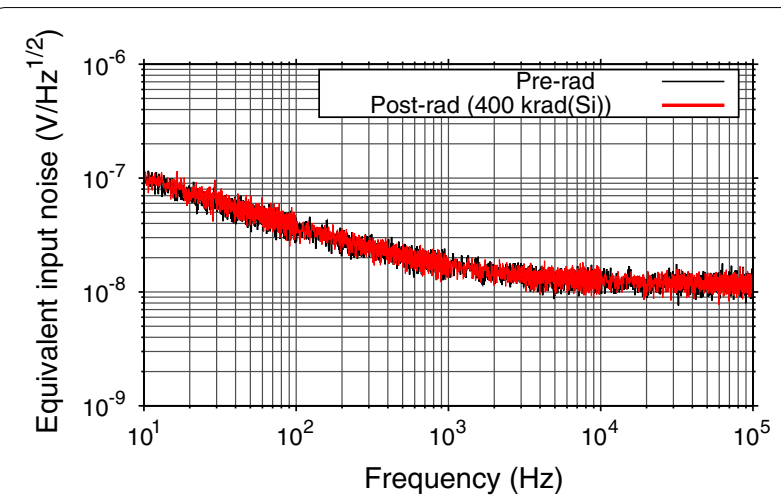

Fig. 9 Measurement results for the equivalent input noise voltage of the ASIC preamplifier under radiation testing

north-to-south direction. It is assumed that a sounding rocket is launched from a horizontal distance of $-260 \mathrm{~km}$ on the ground toward magnetic south with a constant elevation angle of $63.43 \mathrm{deg}$ along the magnetic meridian plane. The horizontal distance is approximately $260 \mathrm{~km}$ toward south from Hagane-yama Station to Uchinoura Space Center, which is the Japanese sounding rocket launch facility. It is planned that the MSEE sensor probe onboard the sounding rocket will measure an LF standard electromagnetic wave during the ascent and be separated from the sounding rocket at maximum altitude. Figure 12 shows the intensities of the LF standard electromagnetic waves and their polarization $(-1=\mathrm{L}$ mode, $0=$ linear, and $+1=\mathrm{R}$ mode) along the assumed trajectory during the ascent for daytime (panels $a, b$, and c) and nighttime (panels d, e, and f). Clearly, the linear polarization in the free space mode turned into whistler-mode waves in the ionosphere. The magnetic field intensity is about $10 \mathrm{pT}$ in free space regardless of the time of day or night, but the intensity in the ionosphere for the nighttime is about 4 


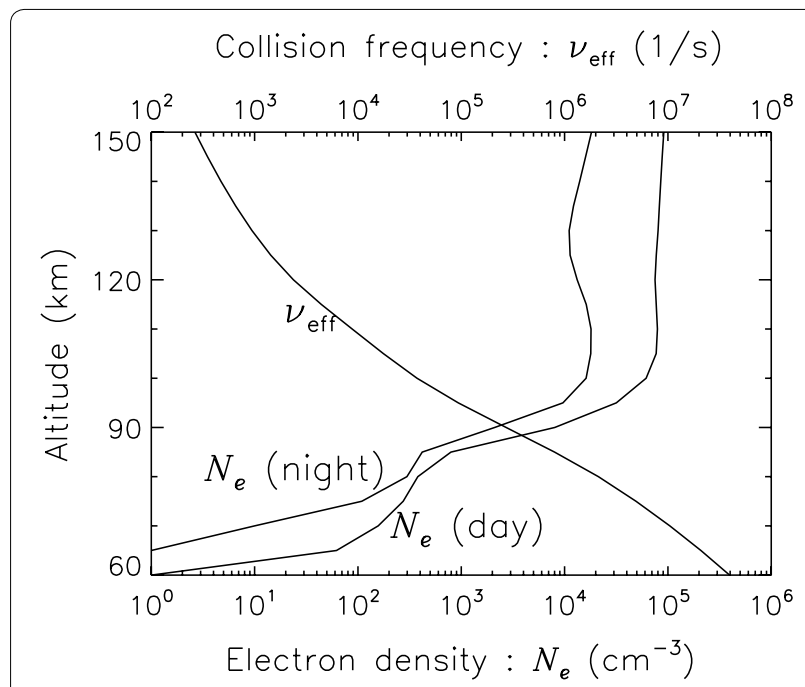

Fig. 10 Altitude profiles of electron density and effective collision frequency in the ionosphere

times stronger than that for the daytime. The NEMI for the ASIC preamplifier combined with the loop antenna is $20 \mathrm{fT} / \mathrm{Hz}^{1 / 2}$ at a frequency of $60 \mathrm{kHz}$, which is sufficiently low for the measurement of LF standard electromagnetic waves. The gain of the ASIC preamplifier combined with the loop antenna is $2 \mathrm{~dB} / \mathrm{nT}$ at a frequency of $60 \mathrm{kHz}$. Then, the output voltages for the LF standard electromagnetic waves $\left(B_{x}\right.$ or $B_{y}$ ) become $130 \mathrm{mV}$ in free space and 0.31 to $1.6 \mathrm{mV}$ in the ionosphere, which are lower than the saturated level of the ASIC preamplifier $(1.5 \mathrm{~V})$. These voltage levels can be detected with a 14-bit AD converter. The temperature change of an MSEE body without a survival heater would be easily several tens of degrees Celsius in a sounding rocket experiment during the ascent. The amplitude variations of the magnetic field along the assumed trajectory include a rapid change (about $3 \mathrm{~dB} / \mathrm{km}$ ) around the boundary of the lower ionosphere. If the temperature difference between on the ground and in the lower ionosphere is $50{ }^{\circ} \mathrm{C}$, the gain variations without/with the BGR circuit may become $2 \mathrm{~dB}$ and less than $1 \mathrm{~dB}$, respectively. Such gain variations directly affect the estimation of the electron density. The temperature compensation technique using the BGR circuit is useful to determine a sharp boundary of the lower ionosphere with a few hundred meters accuracy, which is sufficient for the observation of the ionospheric irregularities having $\mathrm{km}$ scales. Thus, the ASIC preamplifier for a 3-axis loop antenna of the MSEE sensor probe satisfies the electrical performance needed for probing an LF standard electromagnetic wave by a sounding rocket experiment.

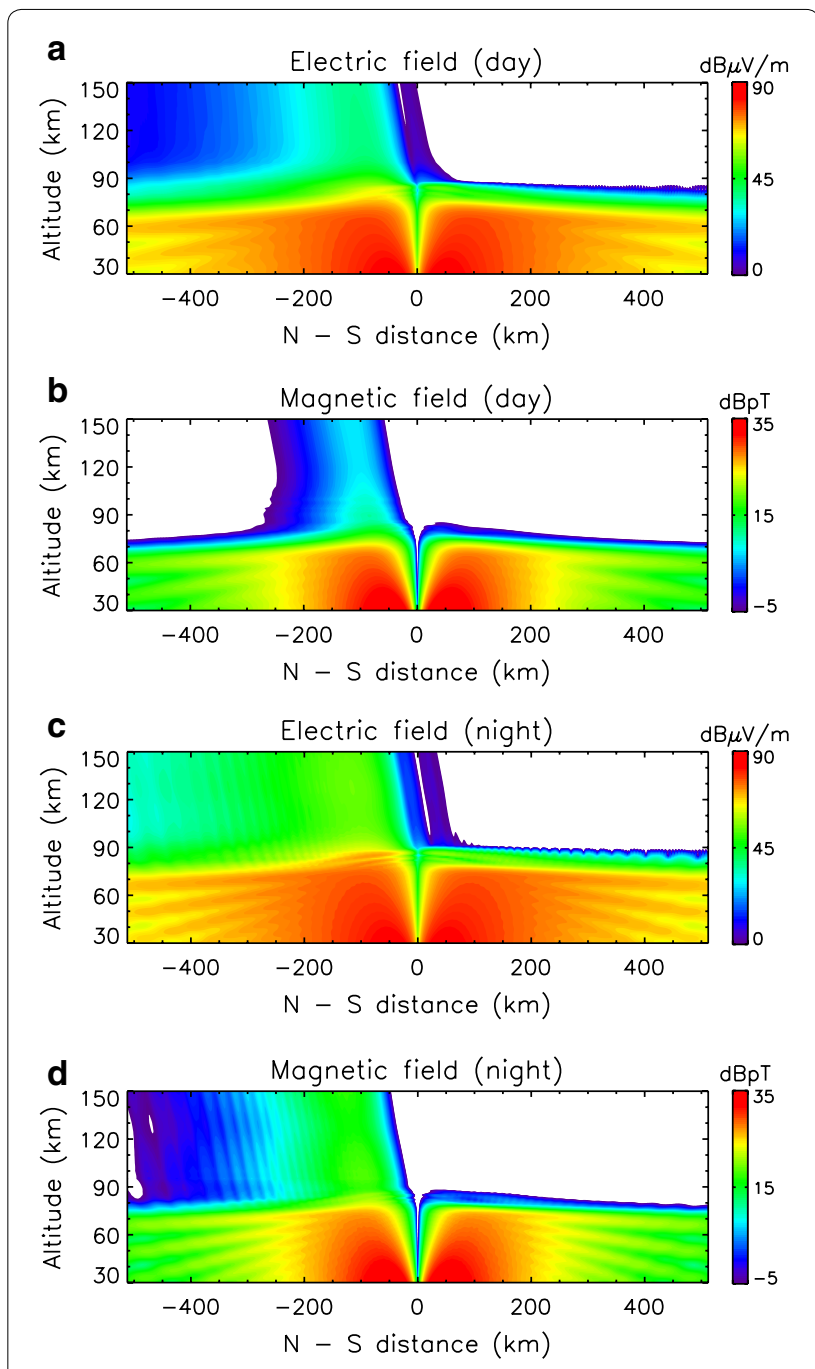

Fig. 11 Intensity distributions of the LF standard electromagnetic waves on the magnetic meridian plane. $\mathbf{a}, \mathbf{b}$ are for the daytime, and c, $\mathbf{d}$ are for the nighttime

\section{Conclusions}

In this study, an ASIC preamplifier for magnetic field observations by an MSEE sensor probe was designed, developed, and assessed. This ASIC preamplifier included low-noise differential amplifiers, gain adjustment amplifiers, and temperature-compensated BGR circuits for a 3 -axis loop antenna in a $5 \times 5 \mathrm{~mm}$ bare chip. The NEMI for the ASIC preamplifier combined with a $0.01-\mathrm{m}^{2}$ loop was adequate for the detection of whistler-mode waves excited by an electron beam from the Space Shuttle (Fig. 7) and an LF standard electromagnetic wave for a virtual sounding rocket experiment (Fig. 12). The ASIC preamplifier showed a high resistance to temperature change (Fig. 8) and TID radiation (Fig. 9). Fundamental 


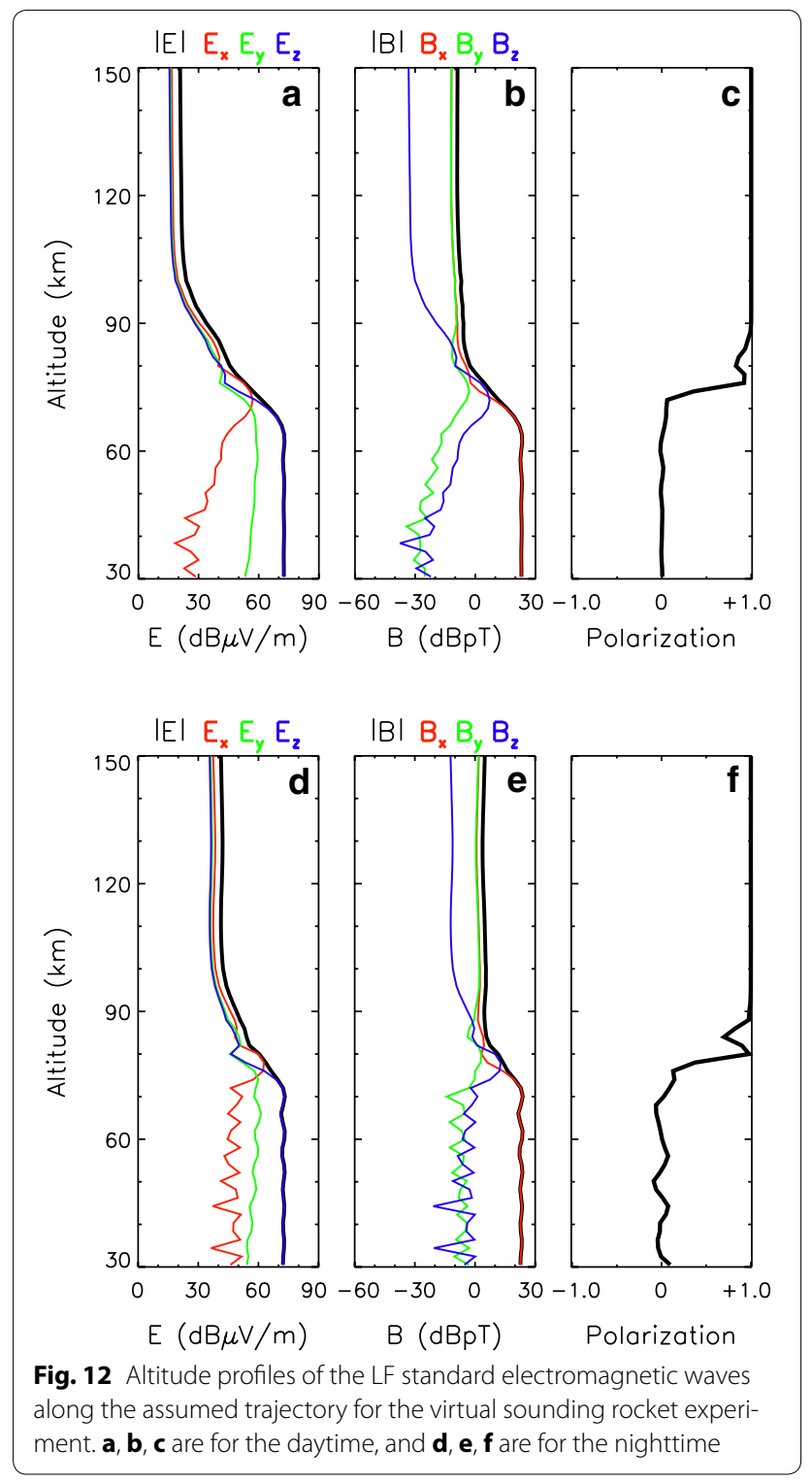

temperature and radiation issues on the miniaturization of MSEE sensor probe were solved by using our developed ASIC preamplifier. A distributed MSEE sensor network motivates separating spatial and temporal variations of plasma phenomena. One remaining issue is the evaluation of the threshold for latch-up caused by $\mathrm{MeV}$ level particles and to embed a latch-up protection circuit in the chip. Furthermore, a chopper stabilization technique (e.g., Yan et al. 2014) will be considered to markedly improve the flicker noise characteristics of the ASIC preamplifier in a future ASIC design. Finally, a major goal of the MSEE sensor probes is to reveal the plasma wave turbulence caused by space missions.

\section{Authors' contributions}

$\mathrm{MO}$ carried out the ASIC design, proposed the temperature compensation method, assisted with all electrical performance, temperature, and radiation tests, and drafted the manuscript. SY participated in the discussion and contributed to the basic design of the loop antenna. HK conceived the idea of the MSEE and helped to draft the manuscript. TZ, KT, HK, and YT all helped with the electrical performance tests. All authors read and approved the final manuscript.

\section{Author details}

${ }^{1}$ Kanazawa University, Kakuma-machi, Kanazawa 920-1192, Japan. ${ }^{2}$ Research Institute for Sustainable Humanosphere, Kyoto University, Gokasho,

Uji 611-0011, Japan. ${ }^{3}$ Panasonic System Networks R\&D Lab. Co., Ltd., Sainen, Kanazawa 920-0024, Japan.

\section{Acknowledgements}

This study was supported by Grant-in-Aid for Scientific Research (23244097, 15H02136, and 15K18076) from the Japan Society for the Promotion of Science, by a research grant for Exploratory Research on Sustainable Humanosphere Science from Research Institute for Sustainable Humanosphere, Kyoto University, a research grant from Japan Power Academy, and Kanazawa University microsatellite project (Kanazawa-SAT ${ }^{3}$ ). This study was also supported by VLSI Design and Education Center, the University of Tokyo, with the collaboration of Cadence Design Systems, Inc., and Mentor Graphics Corporation. The authors thank Dr. A. Kitagawa of Kanazawa University for his valuable comments. The authors also thank Mr. H. Takano of Meiwa System Company for his efforts in manufacturing the prototype of the MSEE sensor probe. The authors also thank Mr. I. Yoda of Radioisotope Research Center, Tokyo Institute of Technology, for his support and cooperation during the radiation tests.

\section{Competing interests}

The authors declare that they have no competing interests.

Received: 1 October 2015 Accepted: 10 May 2016

Published online: 28 May 2016

\section{References}

Bilitza D (2001) International reference ionosphere 2000. Radio Sci 36(2):261275. doi:10.1029/2000RS002432

Cairns IH, Gurnett DA (1991) Plasma waves observed in the near vicinity of the space shuttle. J Geophys Res 96(A8):13913-13929. doi:10.1029/91JA00982

Cao Y, De Cock W, M S, Leroux P (2013) A 4.5 MGy-TID-tolerant CMOS bandgap reference circuit using a dynamic base leakage compensation technique. IEEE Trans Nucl Sci 60(4):2819-2824. doi:10.1109/TNS.2012.2233755

Cattell C et al (2008) Discovery of very large amplitude whistler-mode waves in earth's radiation belts. Geophys Res Lett 35:L01105. doi:10.1029/200 7GL032009

Coillot C, Moutoussamy J, Lebourgeois R, Ruocco S, Chanteur G (2010) Principle and performance of a dual-band search coil magnetometer: a new instrument to investigate fluctuating magnetic fields in space. IEEE Sens $J$ 10(2):255-260. doi:10.1109/JSEN.2009.2030977

Constantinescu OD, Glassmeier K-H, Motschmann U, Treumann RA, Fornaçon K-H, Fränz M (2006) Plasma wave source location using cluster as a spherical wave telescope. J Geophys Res 111:A09221. doi:10.1029/200 5JA011550

Cressler JD, Mantooth HA (2012) Extreme environment electronics. CRC Press, Boca Raton

Fleetwood DM, Eisen HA (2003) Total-dose radiation hardness assurance. IEEE Trans Nucl Sci 50(3):552-564. doi:10.1109/TNS.2003.813130

Fukuhara H, Kojima H, Ishii H, Okada S, Yamakawa H (2012) Tiny waveform receiver with a dedicated system chip for observing plasma waves in space. Meas Sci Technol. doi:10.1088/0957-0233/23/10/105903

Gurnett DA, Kurth WS, Steinberg JT, Banks PM, Bush RI, Raitt WJ (1986) Whistler-mode radiation from the spacelab 2 electron beam. J Geophys Res 13(3):225-228. doi:10.1029/GL013i003p00225 
Hedin AE (1991) Extension of the MSIS thermosphere model into the middle and lower atmosphere. J Geophys Res 96(A2):1159-1172. doi:10.1029/90JA02125

Hjalmarson HP, Pease RL, Witczak SC, Shaneyfelt MR, Schwank JR, Edwards AH, Hembree CE, Mattsson TR (2003) Mechanisms for radiation dose-rate sensitivity of bipolar transistors. IEEE Trans Nucl Sci 50(6):1901-1909. doi:10.1109/TNS.2003.821803

Kojima H, Fukuhara H, Mizuochi Y, Yagitani S, Ikeda H, Miyake Y, Usui H, Iwai H, Takizawa Y, Ueda Y, Yamakawa H (2010) Miniaturization of plasma wave receivers onboard scientific satellites and its application to the sensor network system for monitoring the electromagnetic environments in space. In: Ip W-H, Duldig M (eds) Advances in geosciences vol 21: Solar terrestrial, pp 461-481. World Scientific Publishing, Singapore

Magnes W, Oberst M, Valavanoglou A, Hauer H, Hagen C, Jernej I, Neubauer $\mathrm{H}$, Baumjohann W, Pierce D, Means J, Falkner P (2008) Highly integrated front-end electronics for spaceborne fluxgate sensors. Meas Sci Technol. doi:10.1088/0957-0233/19/11/115801

Martinez-Calderon C, Shiokawa K, Miyoshi Y, Ozaki M, Schofield I, Connors M (2015) Polarization analysis of vif/elf waves observed at subauroral latitudes during the vlf-chain campaign. Earth Planets Space 67:21. doi:10.1186/s40623-014-0178-7

Nagano I, Mambo M, Hutatsuishi G (1975) Numerical calculation of electromagnetic waves in an anisotropic multilayered medium. Radio Sci 10(6):611-617. doi:10.1029/RS010i006p00611

Nagano I, Rosen PA, Yagitani S, Hata M, Miyamura K, Kimura I (1993) Full wave analysis of the Australian omega signal observed by the Akebono satellite. IEICE Trans Commun E76-B(12):1571-1578

Oldham TR, McLean FB (2003) Total ionizing dose effects in MOS oxides and devices. IEEE Trans Nucl Sci 50(3):483-499. doi:10.1109/TNS.2003.812927

Ozaki M, Yagitani S, Kojima H, Takahashi K, Kitagawa A (2014) Current-sensitive CMOS preamplifier for investigating space plasma waves by magnetic search coils. IEEE Sens J 14(2):421-429. doi:10.1109/JSEN.2013.2284011

Ozaki M, Yagitani S, Nagano I, Hata Y, Yamagishi H, Sato N, Kadokura A (2008) Localization of VLF ionospheric exit point by comparison of multipoint ground-based observation with full-wave analysis. Polar Sci 2(4):237-249. doi:10.1016/j.polar.2008.09.001

Ozaki M, Yagitani S, Nagano I, Miyamura K (2009) lonospheric penetration characteristics of elf waves radiated from a current source in the lithosphere related to seismic activity. Radio Sci. doi:10.1029/2008RS003927

Ozaki M, Yagitani S, Takahashi K, Nagano I (2013) Dual-resonant search coil for natural electromagnetic waves in the near-earth environment. IEEE Sens J 13(2):644-650. doi:10.1109/JSEN.2012.2224863
Paulus C, Brederlow R, Kleine U, Thewes R (2001) An efficient and precise design method to optimize device areas in mismatch and flicker-noise sensitive analog circuits. In: Proceedings of ICECS, pp 107-111

Rhouni A, Sou G, Leroy P, Coillot C (2013) Very low 1/f noise and radiationhardened CMOS preamplifier for high-sensitivity search coil magnetometers. IEEE Sens J 13(1):159-166. doi:10.1109/JSEN.2012.2211347

Schrimpf RD, Fleetwood DM (2004) Radiation effects and soft errors in integrated circuits and electronic devices. World Scientific Publishing, Singapore

Sordo-lbáñez S, Piñero-García B, Muñoz-Díaz M, Ragel-Morales A, CeballosCáceres J, Carranza-González L, Espejo-Meana S, Arias-Drake A, RamosMartos J, Mora-Gutiérrez JM, Lagos-Florido MA (2015) A front-end ASIC for a 3-d magnetometer for space applications by using anisotropic magnetoresistors. IEEE Trans Magn. doi:10.1109/TMAG.2014.2356976

Torbert RB, Russell CT, Magnes W, Ergun RE, Lindqvist P-A, LeContel O, Vaith $H$, Macri J, Myers S, Rau D, Needell J, King B, Granoff M, Chutter M, Dors I, Olsson G, Khotyaintsev YV, Eriksson A, Kletzing CA, Bounds S, Anderson B, Baumjohann W, Steller M, Bromund K, Le G, Nakamura R, Strangeway RJ, Leinweber HK, Tucker S, Westfall J, Fischer D, Plaschke F, Porter J, Lappalainen K (2014) The FIELDS instrument suite on MMS: scientific objectives, measurements, and data products. Space Sci Rev. doi:10.1007/ S11214-014-0109-8

Velazco R, Fouillat P, Reis R (2007) Radiation effects on embedded systems. Springer, Dordrecht

Voldman SH (2007) Latchup. Wiley, Chichester

Yagitani S, Nagano I, Miyamura K, Kimura I (1994) Full wave calculation of elf/ vlf propagation from a dipole source located in the lower ionosphere. Radio Sci 29(1):39-54. doi:10.1029/93RS01728

Yagitani S, Ozaki M, Kojima H (2011) A compact loop antenna system for monitoring local electromagnetic environments in geospace. IEICE Trans Commun E94-B(6):1744-1747

Yan B, Zhu W, Liu L, Liu K, Fang G (2014) Equivalent input magnetic noise analysis for the induction magnetometer of $0.1 \mathrm{mhz}$ to $1 \mathrm{hz}$. IEEE Sens J 14(12):4442-4449. doi:10.1109/JSEN.2014.2336971

Zushi T, Kojima H, Onishi K, Ozaki M, Yagitani S, Shimizu S, Yamakawa H (2015) Small sensor probe for measuring plasma waves in space. Earth Planets Space 67:127. doi:10.1186/s40623-015-0298-8

\section{Submit your manuscript to a SpringerOpen ${ }^{\circ}$ journal and benefit from:}

- Convenient online submission

- Rigorous peer review

- Immediate publication on acceptance

- Open access: articles freely available online

- High visibility within the field

- Retaining the copyright to your article

Submit your next manuscript at springeropen.com 